Published in final edited form as:

Gastrointest Endosc Clin N Am. 2009 April ; 19(2): 233-242. doi:10.1016/j.giec.2009.02.008.

\title{
Reflectance Spectroscopy
}

\author{
Michael B. Wallace, MD, \\ Division of Gastroenterology, Mayo Clinic, 4500 San Pablo Road, Jacksonville, Florida, \\ Wallace.Michael@mayo.edu
}

Adam Wax, PhD, Dept. of Biomedical Engineering, Duke University, Durham, NC 27708, A.Wax@duke.edu

David N. Roberts, MD, and

University of Oklahoma Digestive Diseases Section, 920 Stanton L Young Blvd, WP 1360, Oklahoma City, OK 73013, David-Roberts@ouhsc.edu

Robert N. Graf

Dept. of Biomedical Engineering, Duke University, Durham, NC 27708, A.Wax@duke.edu

\section{Introduction}

Reflectance spectroscopy is an emerging technology among the new optically based endoscopic techniques. Providing a rapid and safe evaluation of tissue for dysplasia and ischemia without excision, it overcomes many limitations in histopathologic processing and diagnosis. Reflectance spectroscopy is a form of point-probe technology having the advantages of ease of passage through the accessory channel of a standard diagnostic endoscope and predictable geometry between fibers that provide the light source and those that deliver collected light to the detector. It quantitatively measures the color and intensity of reflected light. Unlike autofluorescence spectroscopy, the reflected light maintains the same wavelength, although different wavelengths are absorbed and reflected to different degrees.

A standard example is oxygenated hemoglobin which, when illuminated with white light, absorbs much of the blue light, and reflects back the red light, giving its characteristic color. Deoxygenated hemoglobin absorbs a higher degree of red light, appearing bluer when illuminated with white light. Reflectance spectroscopy provides information about tissue hemoglobin concentrations and oxygenation status. With the inherent property of malignant tissue to promote angiogenesis, reflectance spectroscopy may be capable of detecting neoplastic tissue based on hemoglobin absorption parameters.

Light scattering spectroscopy is a form of reflectance spectroscopy which utilizes the properties of elastic scattering inherent to structures within the tissues. It measures the extent to which the angular paths of light are altered by structures such as nuclei, mitochondria, and collagen networks. Described herein, further advancements of these principles have provided other potential tools for diagnostic purposes.

\section{Background}

Light reflected from tissue is modified by the processes of absorption and scattering (Figure 1). Absorption of light in the body occurs at specific wavelengths which depend on the biochemical makeup of the probed tissue. In comparison, light scattering produces changes in the trajectory of incident light and affects all wavelengths across the visible spectrum to varying degrees. Light scattering in tissue is determined by the micron scale morphology of the probed sample. The interplay of these two processes, absorption and scattering, provides access to a rich source of information for assessing the health status of tissues. By examining the 
wavelength of reflected light, information about tissue absorption can be obtained. Light scattering, on the other hand, provides information about the way that photons propagate through tissues, providing structural knowledge of the morphology within. Like absorption, scattering information can be obtained by examining the spectral dependence of detected light. After accounting for absorption, the wavelength specific intensities of scattered light which contain unique scattering signatures can be analyzed. Quantitative morphological information about the probed tissue can be obtained through more sophisticated analysis of the angular, polarization, and wavelength dependence of the scattered light.

Absorption of light occurs when the energy of a photon is absorbed and converted to heat energy. Tissue absorption is characterized using the Beer-Lambert law of exponential attenuation with photon path length. The absorption coefficient $\mu$ a, is generally wavelength dependent for biochemicals of interest. The absorption coefficient depends on the concentration and molar absorptivity of biochemicals present in the tissue. The most common tissue absorber is hemoglobin which has a characteristic absorption spectrum. Oxygenated and de-oxygenated hemoglobin preferentially absorb light of different wavelengths, as evidenced by the more reddish color or oxygenated blood and the more bluish color of deoxygenated blood. The extent of absorption by each type of hemoglobin provides information about their relative concentrations, and indirectly provides information about the vascularity and metabolic status of tissue. This is the principal behind optical pulse oximeters which perform an extremely simplistic version of reflectance spectroscopy. Hemoglobin absorption can be used as an indicator for many disease states. For example, malignant and pre-malignant gastrointestinal tissue tends to be more vascular (with higher concentrations of hemoglobin) and also more metabolically active (and thus a higher ratios of deoxy:oxyhemoglobin). Tissue which is also poorly perfused, such as in chronic intestinal ischemia, can also be detected using reflectance spectroscopy, in this case with lower levels of hemoglobin and higher ratios of deoxy:oxyhemoglobin.

Light scattering spectroscopy is a type of reflectance spectroscopy that determines tissue structures by examining elastic scattering. Elastic light scattering is a process in which the illumination light does not undergo a shift in wavelength during the scattering event. Each wavelength of the illumination light is scattered to a different degree by dense structures in the tissue such as nuclei. A portion of the light incident on the tissue is backscattered, or scattered directly in the backwards direction, and can be collected by the fiber optic probe that delivered the light to the tissue or a distinct collection optical fiber. Since no energy is lost during these scattering events, and therefore no wavelength shift occurs, this type of spectroscopy is termed "elastic." By analyzing the varying degree to which individual wavelengths are backscattered across a spectral range, it is possible to determine the size, number, and optical density of the dense structures within a tissue. This light scattering information can be utilized to detect developing abnormalities in a tissue. For example, neoplastic tissue, which is characterized by enlarged nuclei, can be accurately detected at its earliest stages by elastic scattering spectroscopy.

\section{Equipment for measuring reflectance}

A key advantage of using reflectance spectroscopy as a method for diagnosing tissue health is the wide availability and ease of use of the optical instrumentation. The telecom boom of the late 1990's lead to the availability of many off-the-shelf fiber optic components which have been adapted for biomedical spectroscopy by the new field of biophotonics. Further advances in computing power, data transfer and optical instrumentation have further fueled advances in this field. Reflectance spectroscopy systems for biomedical applications contain three key components: a light source, a fiber optic probe, and a spectrometer. It is the particular specifications chosen for of each of these components, along with the methods of analyzing 
the collected data, which suit particular reflectance spectroscopy systems to certain clinical applications.

The light source of a reflectance spectroscopy system is selected primarily based on its brightness and wavelength range. The brightness, or power output, of a light source must be high enough that the reflected signal is sufficiently stronger than the background noise signal such that high fidelity measurements can be made on reasonable time scales. When performing reflectance spectroscopy in vivo, the power of the light incident on tissue must also conform to the maximum power level allowable by ANSI standards so as not to damage the tissue. Coherent light sources, such as lasers and superluminescent diodes, typically illuminate tissue with significantly higher power than white-light arc lamp sources, especially when delivered through optical fibers. While increased brightness offers the potential of a better signal-to-noise ratio, power levels must be carefully measured to prevent thermally damaging the probed tissue.

The wavelength range of a given light source is also typically tailored to a particular application. Some light sources, such as white-light arc lamps, span the entire visible spectrum and extend into the ultraviolet and near-infrared ranges but may present undesirable features such as sharp spectral peaks and substantial infrared components which can heat the tissue. Coherent light sources, however, span a smaller range from a single wavelength up to several tens of nanometers. In the case of a small wavelength range, a light source whose illumination includes the most diagnostically relevant wavelengths for a particular application should be selected. For broadband light sources, it is important to ensure that all the included wavelength components are transmitted satisfactorily through the optical system, avoiding dispersion effects.

The second major component of a reflectance spectroscopy system is a fiber optic probe. The fiber probe both delivers excitation light to the tissue of interest and collects the reflected signal. The use of thin, flexible fiber optics allow light to be delivered to almost any hollow organ of the body. The probes are typically designed to work seamlessly with the auxiliary port of standard endoscopes. The specific geometry and design of the probe can be tailored to meet the specific needs of the application. For example, the degree of tissue penetration and the angle of interface with tissue can be customized by refining the fiber probe geometry. Illumination and signal collection can be accomplished with a single fiber or with a series of fibers grouped in a bundle depending on the intended application.

The spectrometer is the final major component of a reflectance spectroscopy system. Spectrometers split the detected signal into individual wavelength components and detect their respective intensities using a CCD chip or other multi-channel detector. The size and design of a spectrometer dictate the spectral resolution and the range of detectible wavelengths. These specifications have a tradeoff relationship - that is, an increase in spectral resolution results in a decrease in wavelength range and vice versa. Modern spectrometers are available in compact footprints that are ideal for clinical applications. Most systems interface with computers via USB which permit high speed acquisition control and transfer of data. Commercial spectrometers can be controlled via basic, easy-to-use commercial software packages or via customized software which allows for more sophisticated data analysis which can often be performed in real or near real-time.

While the traditional instrument used in reflectance spectroscopy is the spectrometer, advances in spectroscopic techniques have enabled better tissue characterization. We will discuss recent developments in optical methods and advantages of novel fiber probe geometries as they relate to clinical application. A significant influence on the usefulness of reflected light for tissue diagnosis is the method of analysis. Although empirical methods can be used to diagnose 
diseased tissues, advances in physical modeling of the interaction between light and tissues have lead to improved methods for tissue analysis. By linking spectral features to specific tissue characteristics, novel analysis models have provided more detailed information on tissue composition. We will discuss how the use of advanced physical models can provide superior diagnostic capability.

\section{Clinical Applications}

Reflectance spectroscopy techniques have shown important applications in key clinical settings including gastrointestinal ischemia, dysplasia, and neoplasia. Although in infancy of use, these techniques have shown tremendous potential in early studies for widespread application.

Multiple forms of radiographic imaging are available to evaluate for mesenteric ischemia. Acute mesenteric ischemia is often clinically diagnosed early on based on clinical history and symptomatic presentation. On the other hand, chronic mesenteric ischemia can be much more difficult to diagnose with some patients lacking classic symptoms and others presenting with non-specific abdominal pain. Mesenteric angiography, duplex ultrasonography, computerized tomographic angiography, and magnetic resonance angiography all provide excellent forms to evaluate for stenotic lesions to account for symptoms. An endoscopic diagnostic method, however, is potentially advantageous for many patients in that endoscopic evaluation is often performed early on in the diagnostic workup for abdominal pain. Quantitative measurements of mucosal capillary hemoglobin oxygen saturation would also provide an objective, reproducible means to assess the degree of ischemia before and after any intervention.

A fiberoptic catheter-based visible light spectroscopy oximeter was developed for the purpose of continuously measuring mucosal tissue oxygen saturation and has been evaluated for proof of concept in colonic mucosa before and after submucosal epinephrine injection and strangulation of polyp stalks by endoloop in normal volunteers. Oxygen saturations at baseline, prior to manipulation, were between $60-80 \%$. When altered by injection or strangulation, desaturations of the mucosal stalk occurred rapidly down to $18-36 \%$. (1)

This was further demonstrated in three patients with abdominal pain, weight loss, and medical risk factors for chronic mesenteric ischemia. Baseline oximetry measurements were obtained from 25-30 healthy controls with normal mucosa in the esophagus, stomach, duodenum/ jejunum, and colon/rectum. The three patients evaluated had clear evidence of mucosal abnormalities ranging from cyanotic, friable, and erythematous to diffuse erosions and ulcerations. A statistically significant difference in tissue saturation was demonstrated in different segments of the small bowel between the healthy patients and the 3 cases prior to intervention ( $\mathrm{p}=0.003$ ). One patient had an increase in proximal jejunum saturation from $30 \%$ to $60 \%$ after vascular intervention. Another had dramatic improvement in the duodenum (19\% to $51 \%$ ) and proximal jejunum ( $42 \%$ to $56 \%$ ) following intervention. The third had changes from $16 \%$ to $59 \%$ in the proximal jejunum, and mid-duodenal measurements improved from $45 \%$ to $55 \%$ with resolution of gastric ulcers and mottling within three days. Aside from a small patient study size, the technique has limitations in potential sampling error, difficulty reaching the mucosal area of injury, and the likelihood that patients with milder ischemic changes would expectantly have oximetry values much closer to normal. This technique does have other potential applications, however, in the evaluation of intestinal ischemia at surgical anastomoses and post-operative strictures. (2)

Light scattering spectroscopy (LSS) has been shown for many years to have potential use in the detection of dysplasia and carcinoma in situ in a variety of organ system and epithelial cell types including transitional cell epithelium of the bladder, squamous lining of the oral cavity, and columnar cells of the colon and Barrett's esophagus. The nuclear-size distribution and 
refractive index were determined, allowing for quantification of nuclear enlargement, crowding, and hyperchromasia. (3)

In evaluation of patients with Barrett's esophagus, thirteen consecutive patients were analyzed endoscopically with use of light-scattering spectroscopy. White light spectroscopy was utilized to assess the size distribution of cell nuclei. These data were extrapolated to determine the percentage of enlarged nuclei and degree of nuclear crowding. Dysplasia was assigned if more than $30 \%$ of the nuclei exceeded $10 \mu \mathrm{m}$ and histological agreement with 4 blinded pathologists. The sensitivity and specificity for LSS in detecting either high grade dysplasia (HGD) or low grade dysplasia (LGD) were $90 \%$ and $90 \%$ respectively. All HGD samples were properly characterized as such by LSS and $87 \%$ of LGD lesions classified correctly. (4)

In a study of the rat esophagus carcinogenesis model, the angle-resolved low coherence interferometry $(\mathrm{a} / \mathrm{LCI})$ light scattering technique was applied to detecting nuclear morphology changes due to neoplastic transformation (Figure 2). In this approach the unique coherence gating capability of this technique technique was used to isolate light scattered exclusively from the basal layer of the epithelium. The angular distribution of scattered light was used to detect changes in the average size of the cell nuclei in response to treatment with the carcinogen NMBA. Logistic regression revealed that nucleus size was a powerful indicator of precancerous lesions, yielding $80 \%$ sensitivity and $100 \%$ specificity (5). Significantly, this study also revealed chemopreventive action by detecting contracted cell nuclei identified as apoptotic. A follow up study using a/LCI employed the decision line from the first study to prospectively grade tissue samples from this animal model at multiple time points. The technique was found to have high sensitivity (91\%) and specificity (97\%) for detecting precancerous lesions in this model(6).

In analyzing models of colon carcinogenesis, four-dimensional elastic light-scattering spectroscopy (4D-ELF), a type of light scattering spectroscopy was applied to examine the colonic mucosa from rats which were induced toward dysplastic transformation using the carcinogen azoxymethane (AOM). The light scattering data were analyzed using 4D-ELF to determine if preneoplastic changes could be detected. Forty-eight rats were randomized to weekly injections of saline or AOM for two doses. The animals were harvested from 2-20 weeks after the second injection for colon mucosal evaluation. A subset of rat colons was further assessed for aberrant crypt foci (ACF). Induction of ACF by AOM became apparent within 4-6 weeks of the second injection, were predominantly located in the distal colon, and continued to increase over time. Notably, even prior to development of ACF, at two week analysis, significant changes were present in the 4D-ELF fingerprint signatures.(Figure 3) These microarchitectural abnormalities are important in carcinogenesis as they correlate temporally and spatially with subsequent ACF. These detectable alterations in 4D-ELF signatures suggest the presence of a field effect, a feature strongly desirable in early carcinoma detection. (7)

4D-ELF was further utilized to evaluate the superficial blood content in colonic mucosa of Fischer rats induced toward neoplastic changes with AOM. The depth of focused analysis involved the pericryptal capillary plexus and demonstrated significant, reproducible differences between control and pre-neoplastic mucosa. Also, multiple intestinal neoplasias (MIN) mice were evaluated with 4D-ELF for red blood cell content in the small bowel and colonic mucosa. These mice possess a germline mutation in the APC tumor suppressor gene which results in small bowel polyps and neoplasia. As expected, prior to neoplastic transformation there was a marked fingerprinting change in the small intestine but not in the colonic mucosa. Subsequently, a pilot study in the normal appearing mucosa of the transverse colon in thirty-seven patients undergoing routine colonoscopy was performed to evaluate for early increase in blood supply. 4D-ELF analysis of the mucosa revealed a three-fold increased 
in superficial blood in patients with advanced adenomas defined as an adenoma $\geq 1 \mathrm{~cm}$, with high grade dysplasia, or $>25 \%$ villous component. Importantly, none of the high grade lesions were present in the transverse colon but in the cecum, rectum, and sigmoid colon.(8) Clearly, further studies need to be performed with a larger patient cohort to evaluate if these findings are reproducible to determine if this spectroscopic technique would benefit patients widespread in screening protocols for colon carcinoma.

In the arena of pancreatic adenocarcinoma, 4D-ELF and low-coherence enhanced backscattering (LEBS) technologies have been recently utilized to evaluate normal appearing periampullary duodenal wall biopsies in nineteen patients with pancreatic cancer compared with 32 healthy controls. These samples were taken between 1 and $3 \mathrm{~cm}$ from the ampulla and evaluated histologically by a pathologist to confirm that no dysplasia or neoplasia was present in the tissue specimen. In addition, biopsies were taken in the stomach and $10 \mathrm{~cm}$ distal to the ampulla in nine patients from the malignant cohort and four of the control cohort to evaluate for the presence of any field effect. When a combination of five optical markers was used, all derivatives of 4D-ELF and LEBS, the ability to discern normal control from pancreatic adenocarcinoma occurred with a sensitivity of $95 \%$ and specificity of $91 \%$. Discernment between normal and resectable pancreatic adenocarcinoma with this combination of five optical markers resulted in a sensitivity of $100 \%$ and specificity of $94 \%$. These five optical markers were spectal slope, fractal dimension, LEBS full width-half max (FWHM), and LEBS autocorrelation decay. (Figure 4) ANOVA and correlation analyses were performed to ensure that detected differences were not a product of age or smoking history. The diagnostic performance of this test was not altered by tumor size, stage, or location as interestingly, even body and tail lesions resulted in the same abnormal fingerprinting despite absence of proximity to the ampulla. Efforts to detect any field effect in the distal duodenum and stomach were negative.(9)

\section{Future Directions}

While early approaches to reflectance spectroscopy were based on a simple delivery and collection geometry, new approaches have become more sophisticated and offer the potential for a greater impact. The new advances make use of differential measurements and coherence based approaches to obtain greater discrimination.

Differential optical pathlength spectroscopy has been developed by H.J.C.M. Sternborg from Erasmus University Medical Centre Rotterdam (10-12) and is an extension of traditional reflectance spectroscopy that uses two closely matched collection paths to assess subtle differences in photon migration paths. This approach has been used successfully to detect precancerous lesions in the respiratory tract and could potential impact GI diagnostics in the future.

Two promising technologies from the group of Vadim Backman at Northwestern University use reflectance spectroscopy for assessing cancer risk. The four dimensional elastic light fingerprinting technique uses multiple parameters of light scattering to identify diseased tissues. Preliminary results using animal models show that this approach can be used to identify preneoplastic events. Another approach from this group is the low coherence enhanced backscattering method $(13,14)$. This technology exploits a coherent effect to isolate light scattering with a specific penetration depth. This approach has been applied to assessing risk of colon cancer by examining the uninvolved mucosa and presents significant potential as a future screening technique. This diagnostic tool highlights the presence of field "defects" or dysplasia where, by gross histologic evaluation, tissue is otherwise normal but interrogation by quantifying red cell count and superficial microvascular patterns reveals preneoplastic changes. 
Angle-resolved low coherence interferometry $(5,6,15)$ has been developed by Adam Wax at Duke University as a combination of light scattering and optical coherence tomography. In this modality, depth resolved nuclear morphology measurements are used to assess tissue health. Greater than $90 \%$ sensitivity and specificity for detecting dysplasia have been obtained in ex vivo human tissue studies of Barrett's esophagus tissue (15). In vivo clinical trials are underway to detect pre-cancerous lesions in Barrett's esophagus patients. Preliminary results that use this approach have been presented at the ASGE meeting, indicating high sensitivity and specificity.

\section{Summary}

The integration of these technologies into standard endoscopic systems will provide a rapid, accurate, and dependable avenue to evaluate extensive areas of gastrointestinal tissues. A "heads-up" display on the endoscopic field of view with spectroscopic results of underlying microarchitectural abnormalities will provide for targeting of dysplastic and neoplastic tissue with anticipated greater diagnostic sensitivity, earlier detection in high risk populations, and computed diagnostic information removing the risk of sampling error and subjectivity to endoscopic sampling. This model will also obviate the endoscopist from deciphering spectroscopy results. The current point-probe accessory does limit the amount of tissue that can be realistically evaluated, however, when this limitation is overcome, larger fields of tissue will be screened with an expectant wide variety of applications.

\section{Acknowledgments}

This work was supported by Grant R33-CA109907 from the National Cancer Institute

\section{Abbreviations}

$\begin{array}{ll}\text { LSS } & \text { light scattering spectroscopy } \\ \text { HGD } & \text { high grade dysplasia } \\ \text { LGD } & \text { low grade dysplasia } \\ \text { LEBS } & \text { low-coherence enhanced backscattering } \\ \text { MIN } & \text { multiple intestinal neoplasms } \\ \text { ACF } & \text { aberrant crypt foci } \\ \text { 4D-ELF } & \text { four-dimensional elastic light-scattering spectroscopy } \\ \text { AOM } & \text { azoxymethane } \\ \text { NMBA } & N \text {-nitrosomethylbenzylamine } \\ \text { a/LCI } & \text { angle-resolved low coherence interferometry }\end{array}$

\section{References}

1. Friedland S, Benaron D, Parachikov I, Soetikno R. Measurement of mucosal capillary hemoglobin oxygen saturation in the colon by reflectance spectrophotometry. Gastrointestinal endoscopy 2003;57 (4):492-497. [PubMed: 12665758]

2. Friedland S, Benaron D, Coogan S, Sze DY, Soetikno R. Diagnosis of chronic mesenteric ischemia by visible light spectroscopy during endoscopy. Gastrointestinal endoscopy 2007;65(2):294-300. [PubMed: 17137857]

3. Backman V, Wallace MB, Perelman LT, et al. Detection of preinvasive cancer cells. Nature 2000;406 (6791):35-36. [PubMed: 10894529] 
4. Wallace MB, Perelman LT, Backman V, et al. Endoscopic detection of dysplasia in patients with Barrett's esophagus using light-scattering spectroscopy. Gastroenterology 2000;119(3):677-682. [PubMed: 10982761]

5. Wax A, Yang CH, Muller MG, et al. In situ detection of neoplastic transformation and chemopreventive effects in rat esophagus epithelium using angle-resolved low-coherence interferometry. Cancer Research 2003;63(13):3556-3559. [PubMed: 12839941]

6. Wax A, Pyhtila JW, Graf RN, et al. Prospective grading of neoplastic change in rat esophagus epithelium using angle-resolved low-coherence interferometry. J Biomed Opt 2005;10(5)

7. Roy HK, Liu Y, Wali RK, et al. Four-dimensional elastic light-scattering fingerprints as preneoplastic markers in the rat model of colon carcinogenesis. Gastroenterology 2004;126(4):1071-1081. discussion 948. [PubMed: 15057746]

8. Wali RK, Roy HK, Kim YL, et al. Increased microvascular blood content is an early event in colon carcinogenesis. Gut 2005;54(5):654-660. [PubMed: 15831911]

9. Liu Y, Brand RE, Turzhitsky V, et al. Optical markers in duodenal mucosa predict the presence of pancreatic cancer. Clin Cancer Res 2007;13(15 Pt 1):4392-4399. [PubMed: 17671121]

10. Aerts JG, Bard MP, Amelink A, et al. Oxygen saturation measured in vivo in bronchial tumors during bronchoscopy in patients with lung cancer using differential path length spectroscopy (DPS) correlates with survival, stage of the disease, and expression of HIF1a. Journal of Clinical Oncology 2006;24(18):561S-S.

11. Bard MPL, Amelink A, Skurichina M, et al. Optical spectroscopy for the classification of malignant lesions of the bronchial tree. Chest 2006;129(4):995-1001. [PubMed: 16608949]

12. Amelink A, Sterenborg H, Bard MPL, Burgers SA. In vivo measurement of the local optical properties of tissue by use of differential path-length spectroscopy. Opt Lett 2004;29(10):1087-1089. [PubMed: 15181994]

13. Roy HK, Kim YL, Liu Y, et al. Risk stratification of colon carcinogenesis through enhanced backscattering spectroscopy analysis of the uninvolved colonic mucosa. Clinical Cancer Research 2006;12(3):961-968. [PubMed: 16467111]

14. Kim YL, Turzhitsky VM, Liu Y, et al. Low-coherence enhanced backscattering: review of principles and applications for colon cancer screening. Journal of Biomedical Optics 2006;11(4)

15. Pyhtila JW, Chalut KJ, Boyer JD, et al. In situ detection of nuclear atypia in Barrett's esophagus using angle-resolved low coherence interferometry. Gastrointestinal Endoscopy 2007;65:487-491.

[PubMed: 17321252] 


\section{Incident light}

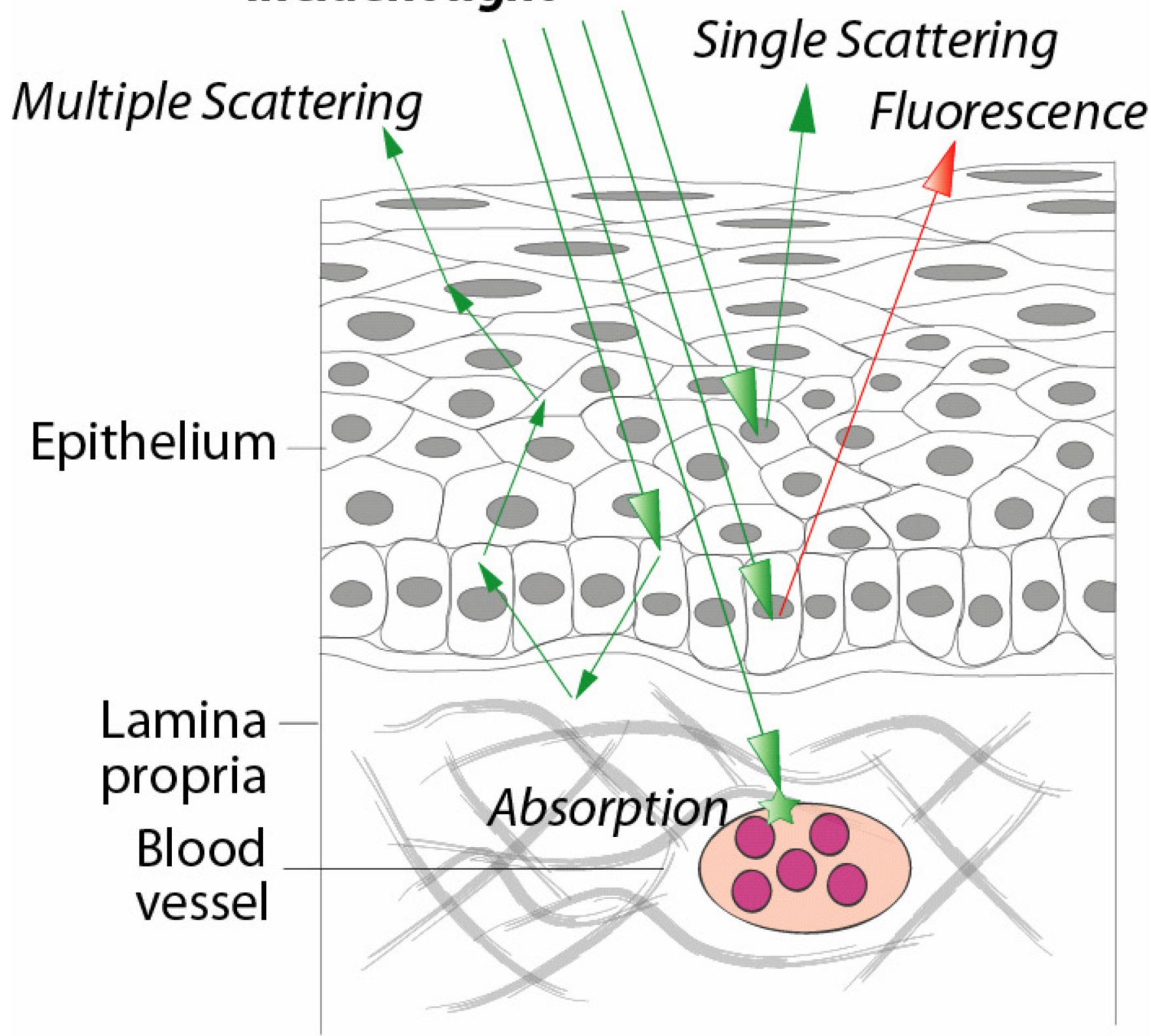

Figure 1. 
(a) Normal
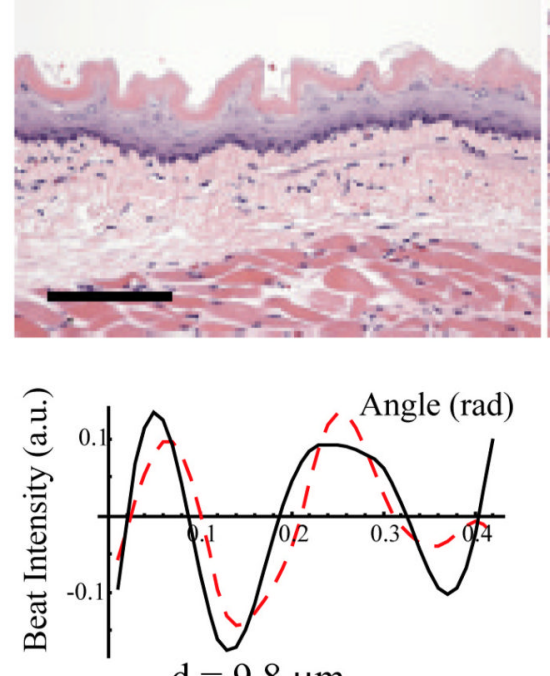

$\mathrm{d}=9.8 \mu \mathrm{m}$

(c) High Grade Dysplasia
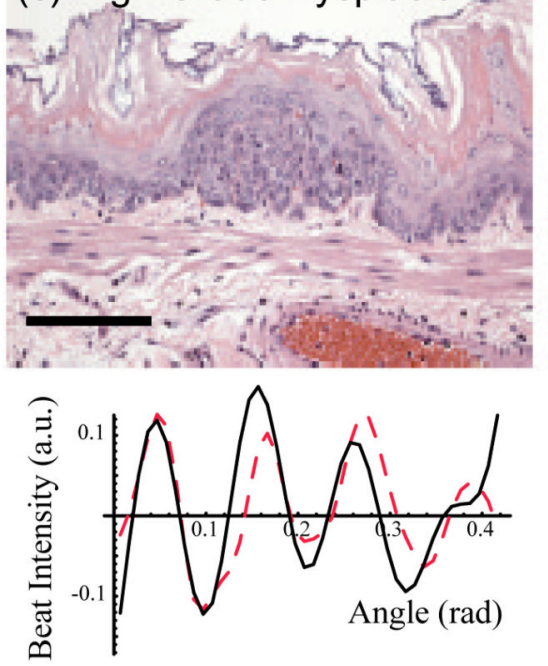

$\mathrm{d}=14.4 \mu \mathrm{m}$ (b) Low Grade Dysplasia
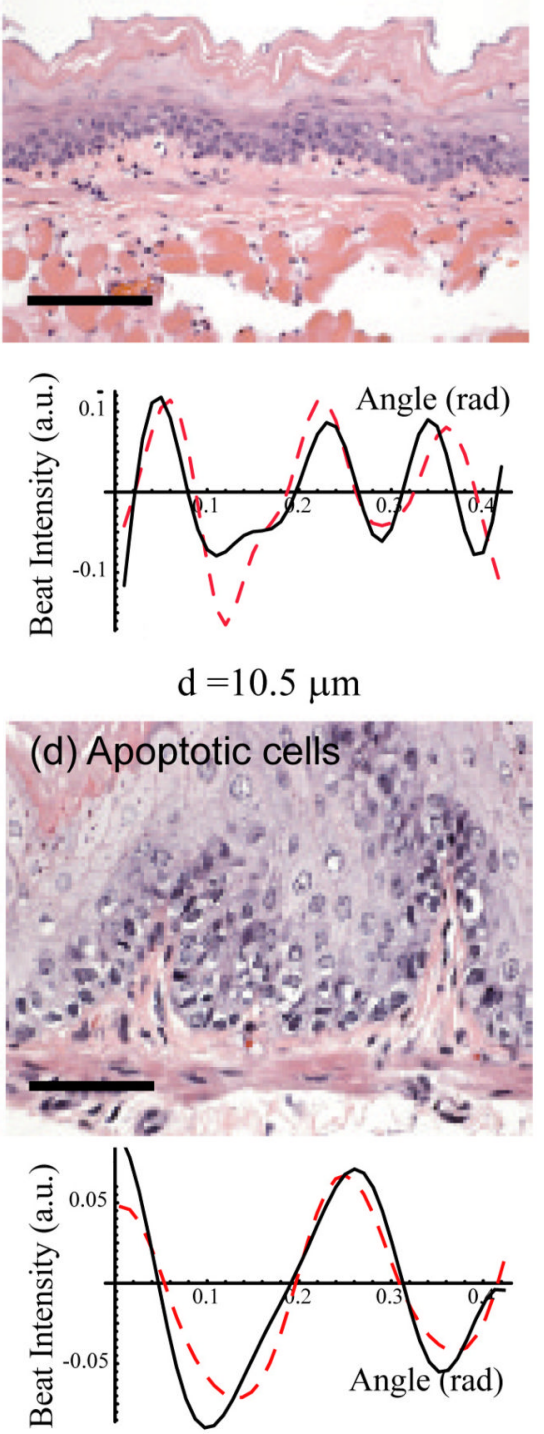

$\mathrm{d}=6.3 \mu \mathrm{m}$ 97\% Specificity (61/63 normal sites)

Figure 2. 

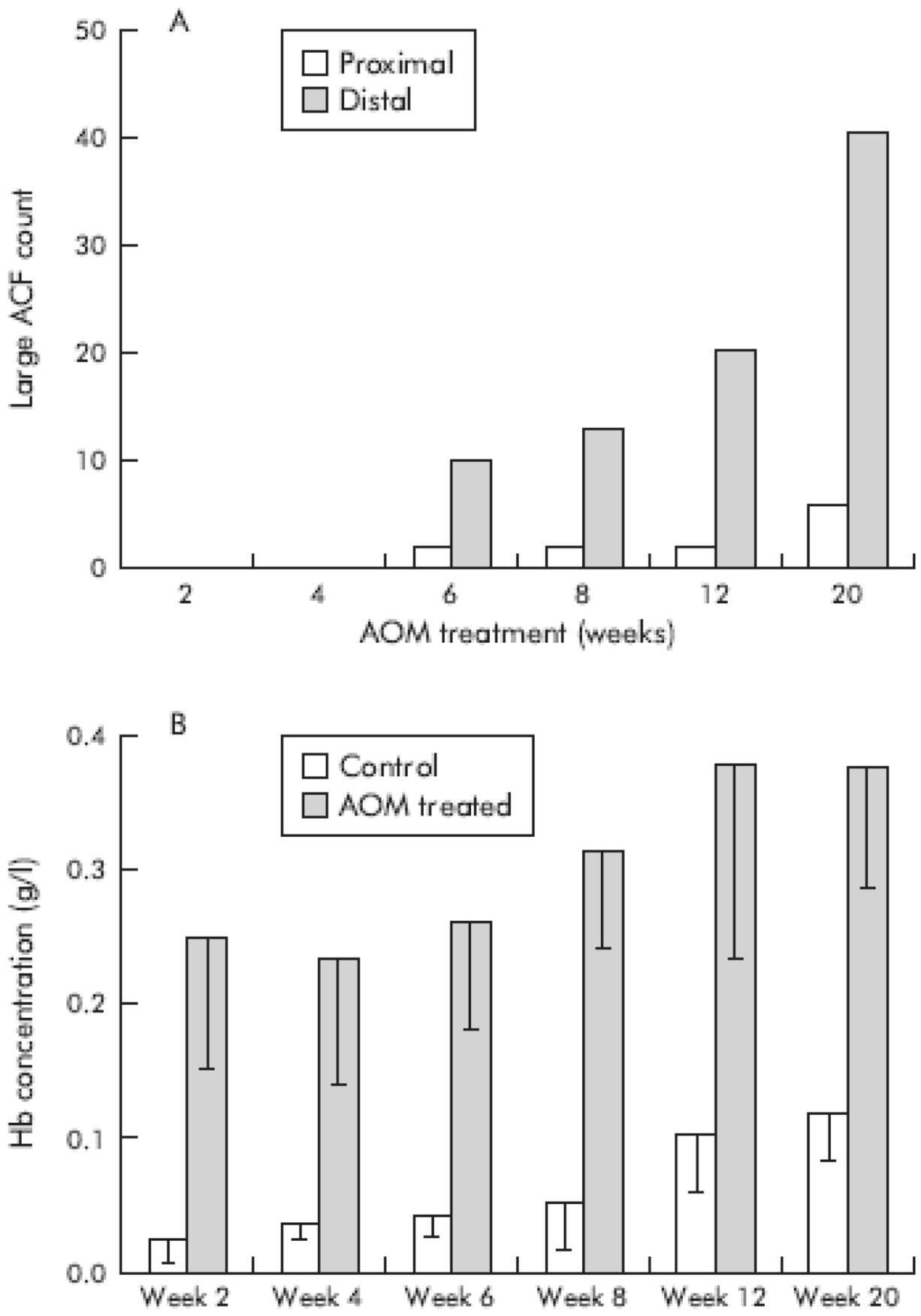

Figure 3. 
Control

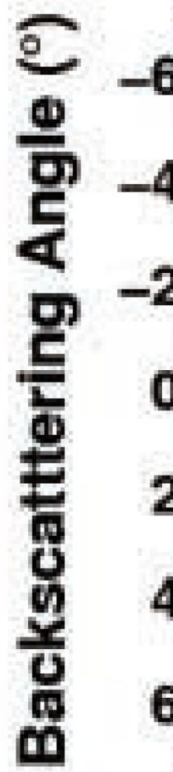

6

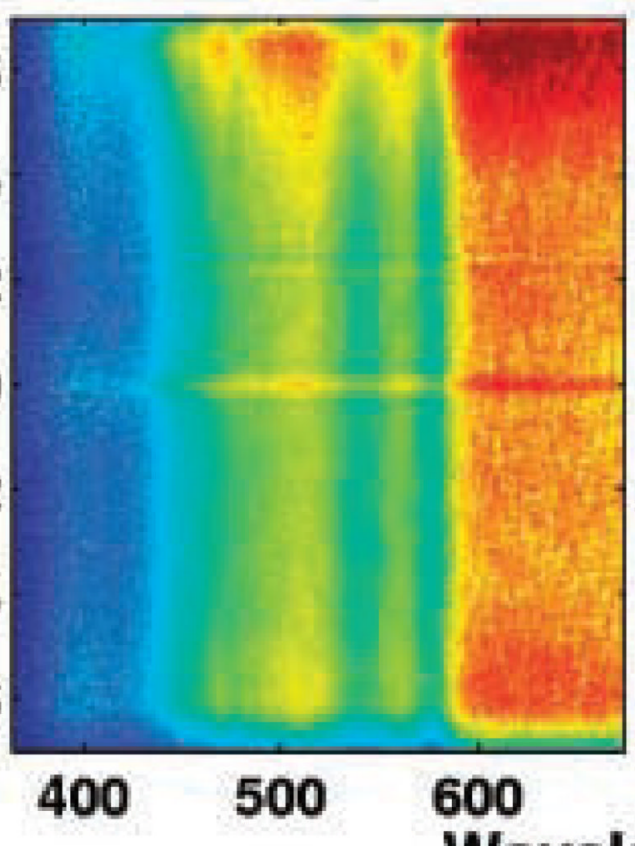

Wavelength (nm)
Pancreatic Cancer

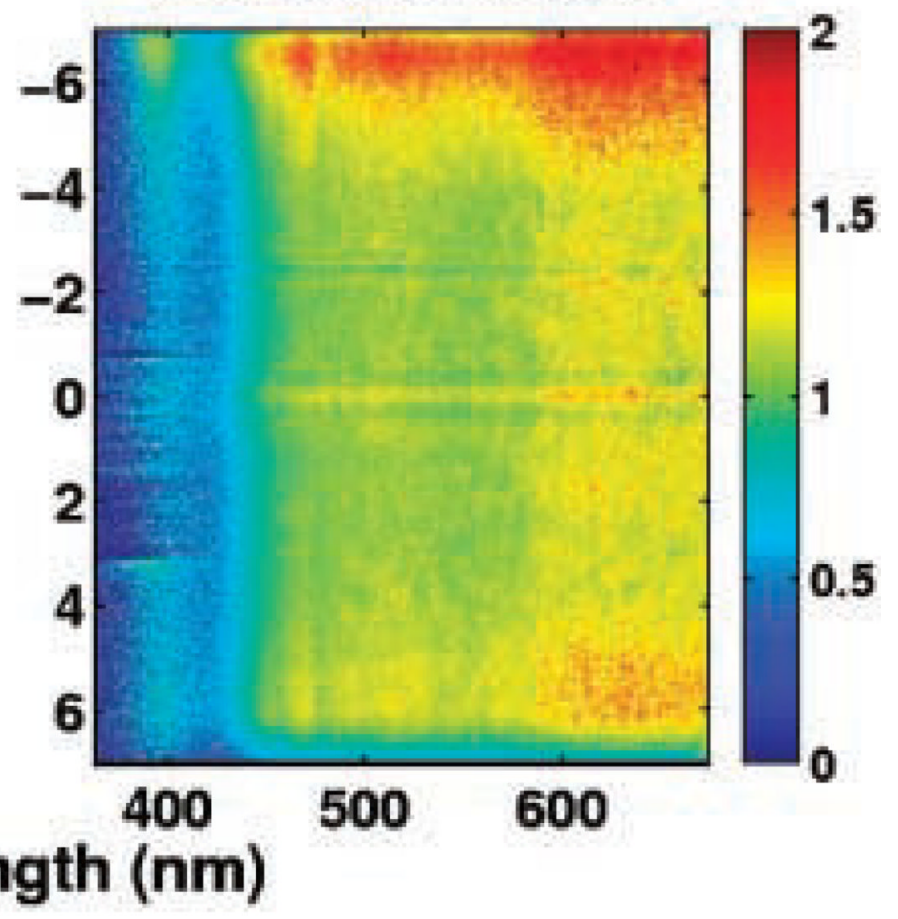

Figure 4. 